\title{
Oscillation Criteria of Even Order Delay Dynamic Equations with Nonlinearities Given by Riemann-Stieltjes Integrals
}

\author{
Haidong Liu and Cuiqin Ma \\ School of Mathematical Sciences, Qufu Normal University, Shandong 273165, China \\ Correspondence should be addressed to Haidong Liu; tomlhd983@163.com
}

Received 7 September 2013; Revised 29 January 2014; Accepted 4 February 2014; Published 16 March 2014

Academic Editor: Ferhan M. Atici

Copyright (C) 2014 H. Liu and C. Ma. This is an open access article distributed under the Creative Commons Attribution License, which permits unrestricted use, distribution, and reproduction in any medium, provided the original work is properly cited.

\begin{abstract}
We study the oscillatory properties of the following even order delay dynamic equations with nonlinearities given by RiemannStieltjes integrals: $\left(p(t)\left|x^{\Delta^{n-1}}(t)\right|^{\alpha-1} x^{\Delta^{n-1}}(t)\right)^{\Delta}+f(t, x(\delta(t)))+\int_{a}^{\sigma(b)} k(t, s)|x(g(t, s))|^{\theta(s)} \operatorname{sgn}(x(g(t, s))) \Delta \xi(s)=0$, where $t \quad \epsilon$ $\left[t_{0}, \infty\right)_{\mathbb{T}}:=\left[t_{0}, \infty\right) \cap \mathbb{T}, \mathbb{T}$ a time scale which is unbounded above, $n \geqslant 2$ is even, $|f(t, u)| \geqslant q(t)\left|u^{\alpha}\right|, \alpha>0$ is a constant, and $\theta:[a, b]_{\mathbb{T}_{1}} \rightarrow \mathbb{R}$ is a strictly increasing right-dense continuous function; $p, q:\left[t_{0}, \infty\right)_{\mathbb{T}} \rightarrow \mathbb{R}, k:\left[t_{0}, \infty\right)_{\mathbb{T}} \times[a, b]_{\mathbb{T}_{1}} \rightarrow \mathbb{R}$, $\delta:\left[t_{0}, \infty\right)_{\mathbb{T}} \rightarrow\left[t_{0}, \infty\right)_{\mathbb{T}}$, and $g:\left[t_{0}, \infty\right)_{\mathbb{T}} \times[a, b]_{\mathbb{T}_{1}} \rightarrow\left[t_{0}, \infty\right)_{\mathbb{\mathbb { T }}}$ are right-dense continuous functions; $\xi:[a, b]_{\mathbb{J}_{1}} \rightarrow \mathbb{R}$ is strictly increasing. Our results extend and supplement some known results in the literature.
\end{abstract}

\section{Introduction}

In this paper, we consider the following even order delay dynamic equations with nonlinearities of the form:

$$
\begin{aligned}
& \left(p(t)\left|x^{\Delta^{n-1}}(t)\right|^{\alpha-1} x^{\Delta^{n-1}}(t)\right)^{\Delta}+f(t, x(\delta(t))) \\
& \quad+\int_{a}^{\sigma(b)} k(t, s)|x(g(t, s))|^{\theta(s)} \operatorname{sgn}(x(g(t, s))) \Delta \xi(s)=0,
\end{aligned}
$$

where $n \geqslant 2$ is even, $t \in\left[t_{0}, \infty\right)_{\mathbb{T}}:=\left[t_{0}, \infty\right) \cap \mathbb{\mathbb { T }}$, and $t_{0} \in \mathbb{T}$, $\mathbb{T}$ is a time scale which is unbounded above, and the following are satisfied:

$\left(H_{1}\right) a, b \in \mathbb{T}_{1}, \mathbb{T}_{1}$ is another time scale, $C_{r d}(\mathbb{D}, \mathbb{S})$ denotes the collection of all functions $f: \mathbb{D} \rightarrow \mathbb{S}$ which are right-dense continuous on $\mathbb{D}$;

$\left(H_{2}\right) p(t) \in C_{r d}\left(\left[t_{0}, \infty\right)_{\mathbb{T}},(0, \infty)\right), p^{\Delta}(t) \geqslant 0, P(t):=$ $\int_{t_{0}}^{t} p^{-1 / \alpha}(s) \Delta s, \lim _{t \rightarrow \infty} P(t)=\infty$, and $\theta(t) \in C_{r d}([a$, $\left.b]_{\mathbb{1}_{1}},(0, \infty)\right)$ is a strictly increasing and satisfying $0<\theta(a)<\alpha<\theta(b), k(t, s) \in C_{r d}\left(\left[t_{0}, \infty\right)_{\mathbb{T}} \times\right.$ $\left.[a, b]_{\mathbb{T}_{1}},[0, \infty)\right)$;

$\left(H_{3}\right) \delta(t) \in C_{r d}\left(\left[t_{0}, \infty\right)_{\mathbb{T}},\left[t_{0}, \infty\right)_{\mathbb{T}}\right), \delta(t) \leqslant t$, for $t \in$ $\left[t_{0}, \infty\right)_{\mathbb{T}}, \lim _{t \rightarrow \infty} \delta(t)=\infty, \delta^{\Delta}(t)>0$ is right-dense continuous on $\left[t_{0}, \infty\right)_{\mathbb{T}}$, and $\widetilde{\mathbb{T}}:=\delta(\mathbb{T}) \subset \mathbb{T}$ is a time scale, $\delta(\sigma(t))=\sigma(\delta(t))$ for all $t \in\left[t_{0}, \infty\right)_{\mathbb{T}}$, where $\sigma(t)$ is the forward jump operator on $\left[t_{0}, \infty\right)_{\mathbb{T}}$;

$\left(H_{4}\right) g(t, s) \in C_{r d}\left(\left[t_{0}, \infty\right)_{\mathbb{T}} \times[a, b]_{\mathbb{T}_{1}},\left[t_{0}, \infty\right)_{\mathbb{T}}\right), g(t, s) \geqslant$ $\delta(t)$, and $\lim _{t \rightarrow \infty} g(t, s)=\infty$, for any $s \in[a, b]_{\mathbb{T}_{1}} ;$

$\left(H_{5}\right) f(t, u) \in C\left(\left[t_{0}, \infty\right)_{\mathbb{T}} \times \mathbb{R}, \mathbb{R}\right)$ is a continuous function such that $u f(t, u)>0$, for all $u \neq 0$ and there exist a positive right-dense continuous function $q(t)$ defined on $\left[t_{0}, \infty\right)_{\mathbb{T}}$ and a constant $\alpha>0$, such that $|f(t, u)| \geqslant$ $q(t)\left|u^{\alpha}\right|$ for all $t \in\left[t_{0}, \infty\right)_{\mathbb{T}}$ and for all $u \in \mathbb{R}$;

$\left(H_{6}\right) \xi:[a, b]_{\mathbb{T}_{1}} \rightarrow \mathbb{R}$ is strictly increasing. $\int_{a}^{\sigma(b)} f(s) \Delta \xi(s)$ denotes the Riemann-Stieltjes integral of the function $f$ on $[a, \sigma(b)]_{\mathbb{T}_{1}}$ with respect to $\xi$.

By a solution of (1), we mean a function $x(t)$ such that $x(t) \in C_{r d}^{n-1}\left[t_{x}, \infty\right)_{\mathbb{T}}$ and $p(t)\left|x^{\Delta^{n-1}}(t)\right|^{\alpha-1} x^{\Delta^{n-1}}(t) \quad \epsilon$ $C_{r d}^{1}\left[t_{x}, \infty\right)_{\mathbb{T}}, t_{x} \geqslant t_{0}$ and satisfying (1) for all $t \geqslant t_{x}$, where $C_{r d}^{1}\left[t_{x}, \infty\right)_{\mathbb{T}}$ denotes the set of right-dense continuously $\Delta$ differentiable functions on $\left[t_{x}, \infty\right)_{\mathbb{T}}$. In the sequel, we restrict our attention to those solutions of (1) which exist on the halfline $\left[t_{x}, \infty\right)_{\mathbb{T}}$ and satisfy $\sup \left\{|x(t)|: t \in[\widetilde{T}, \infty)_{\mathbb{T}}\right\}>0$ for any $\widetilde{T} \geqslant t_{x}$. A nontrivial solution of (1) is called oscillatory if it has arbitrary large zeros; otherwise, it is called nonoscillatory. 
Equation (1) is said to be oscillatory if all its solutions are oscillatory.

If $\alpha$ is a quotient of odd positive integers, $f(t, u)=q(t) u^{\alpha}$, $k(t, s)=0$, then (1) simplifies to the even order dynamic equation

$$
\left(a(t)\left(x^{\Delta^{n-1}}(t)\right)^{\alpha}\right)^{\Delta}+q(t)(x(t))^{\alpha}=0 .
$$

If $f(t, u)=q(t)|u|^{\alpha-1} u, \mathbb{T}_{1}=\mathbb{N}, a=1, b=k$ for $n \in$ $\mathbb{N}$, and $\xi(s)=s ; \theta(s)=\alpha_{s},(s=1,2, \ldots, k)$ satisfying $\alpha_{1}>$ $\alpha_{2}>\cdots>\alpha_{m}>\alpha>\alpha_{m+1}>\cdots>\alpha_{k} ; k(t, s)=p_{s}(t), s=$ $1,2, \ldots, k ; g(t, s)=\delta(t)$, then (1) reduces to

$$
\begin{gathered}
\left(r(t)\left|x^{\Delta^{n-1}}(t)\right|^{\alpha-1} x^{\Delta^{n-1}}(t)\right)^{\Delta}+p(t)|x(\delta(t))|^{\alpha-1} x(\delta(t)) \\
+\sum_{i=1}^{k} p_{i}(t)|x(\delta(t))|^{\alpha_{i}-1} x(\delta(t))=0 .
\end{gathered}
$$

In recent years, more and more people have been interested in studying the oscillatory behavior of higher order dynamic equations on time scales, see [1-13] and references therein. For an introduction to time scale calculus and dynamic equations, we refer the reader to the landmark paper of Hilger [14] and the seminal book by Bohner and Peterson [15] for a comprehensive treatment of the subject. In particular, Grace [9] studied the even order dynamic equation (2). By employing generalized Riccati techniques, he established some new criteria which ensure that (2) is oscillatory. Chen and $\mathrm{Qu}$ [8] investigated the even order advanced type dynamic equation (3). They got some new oscillation criteria for (3) by introducing parameter functions.

In the present paper, we will establish several oscillation criteria for the more general equation (1). Our work is of significance because (1) allows an infinite number of nonlinear terms and even a continuum of nonlinearities determined by the function $\xi$. Our results extend and supplement a number of other existing results and handle the cases which are not covered by known criteria.

\section{Preliminaries}

In the sequel, we denote by $L_{\xi}[a, b]$ the set of RiemannStieltjes integrable functions on $[a, \sigma(b))_{\mathbb{V}_{1}}$ with respect to $\xi$, and we use the convention that $\ln 0=-\infty, e^{-\infty}=0$.

Lemma 1 (Kiguarde's Lemma [16, Theorem 5]). Let $n \in \mathbb{N}$, $f \in C_{r d}^{n}(\mathbb{T}, \mathbb{R})$, and $\sup \mathbb{\mathbb { V }}=\infty$. Suppose that $f$ is either positive or negative and $f^{\Delta^{n}}$ is not identically zero and is either nonnegative or nonpositive on $\left[t_{0}, \infty\right)_{\mathbb{T}}$ for some $t_{0} \epsilon$ $\mathbb{T}$. Then there exist $t_{1} \in\left[t_{0}, \infty\right)_{\mathbb{T}}, m \in[0, n)_{\mathbb{Z}}$ such that $(-1)^{n-m} f(t) f^{\Delta^{n}}(t) \geqslant 0$ holds for all $t \in\left[t_{1}, \infty\right)_{\mathbb{T}}$ with

(i) $f(t) f^{\Delta^{j}}(t)>0$ holds for all $t \in\left[t_{1}, \infty\right)_{\mathbb{T}}$ and all $j \in$ $[0, m)_{\mathbb{Z}}$

(ii) $(-1)^{m+j} f(t) f^{\Delta^{j}}(t)>0$ holds for all $t \in\left[t_{1}, \infty\right)_{\mathbb{T}}$ and all $j \in[m, n)_{\mathbb{Z}}$.
In order to present the next lemma, we use the Taylor monomials (see $\left[15\right.$, section 1.6]) $\left\{h_{n}(t, s)\right\}_{n=0}^{\infty}$ which are defined recursively by

$$
h_{n+1}(t, s)=\int_{s}^{t} h_{n}(\tau, s) \Delta \tau, \quad t, s \in \mathbb{T}, n \geqslant 1
$$

where $h_{0}(t, s)=1$.

Lemma 2 (see [1]). Let $\sup \mathbb{\mathbb { T }}=\infty$ and $f \in C_{r d}^{n}(\mathbb{T}, \mathbb{R})(n \geqslant$ 2). Moreover, suppose that Kigurade's theorem holds with $m \in$ $[0, n)_{\mathbb{Z}}$ and $f^{\Delta^{n}}(t) \leqslant 0$ on $\mathbb{T}$. Then there exists a sufficiently large $t_{1} \in \mathbb{T}$ such that

$$
f^{\Delta}(t) \geqslant h_{m-1}\left(t, t_{1}\right) f^{\Delta^{m}}(t) \quad \forall t \in\left[t_{1}, \infty\right)_{\mathbb{V}} \cdot
$$

Lemma 3 (see [1]). Assume that the conditions of Lemma 2 hold. Then

$$
f(t) \geqslant h_{m}\left(t, t_{1}\right) f^{\Delta^{m}}(t) \quad \forall t \in\left[t_{1}, \infty\right)_{\mathbb{T}}
$$

Lemma 4 (see [17]). Suppose that $\left(H_{3}\right)$ holds. Let $x: \mathbb{T} \rightarrow \mathbb{R}$. If $x^{\Delta}$ exists for all sufficiently large $t \in \mathbb{T}$, then $(x(\delta(t)))^{\Delta}=$ $x^{\Delta}(\delta(t)) \delta^{\Delta}(t)$ for all sufficiently large $t \in \mathbb{T}$.

Lemma 5 (see [15]). Assume that $x(t)$ is $\Delta$-differentiable and eventually positive or eventually negative; then

$$
\left(x^{\alpha}(t)\right)^{\Delta}=\alpha\left\{\int_{0}^{1}[(1-h) x(t)+h x(\sigma(t))]^{\alpha-1} \mathrm{~d} h\right\} x^{\Delta}(t) .
$$

Lemma 6 (see [18]). Suppose $X$ and $Y$ are nonnegative; then

$$
\gamma X Y^{\gamma-1}-X^{\gamma} \leqslant(\gamma-1) Y^{\gamma}, \quad \gamma>1
$$

where equality holds if and only if $X=Y$.

Lemma 7 (see [19]). Let $u(t) \in C_{r d}\left([a, b]_{\mathbb{T}_{1}}, \mathbb{R}\right)$ and $\eta(t) \in$ $L_{\xi}[a, b]$ satisfy $u(t) \geqslant 0(\not \equiv 0), \eta(t)>0$ on $[a, b]_{\mathbb{V}_{1}}$, and

$$
\int_{a}^{\sigma(b)} \eta(s) \Delta \xi(s)=1
$$

Then

$$
\int_{a}^{\sigma(b)} \eta(s) u(s) \Delta \xi(s) \geqslant \exp \left(\int_{a}^{\sigma(b)} \eta(s) \ln [u(s)] \Delta \xi(s)\right) .
$$




\section{Main Results}

Theorem 8. Assume that $\left(H_{1}\right)-\left(H_{6}\right)$ hold. If there exist a function $\phi(t) \in C_{r d}^{1}\left(\left[t_{0}, \infty\right)_{\mathbb{T}},(0, \infty)\right)$ and a function $\eta(t) \in$ $L_{\xi}[a, b]$ satisfying $\eta(s)>0$ on $[a, b]_{\mathbb{T}_{1}}$ and for all $t_{1} \in\left[t_{0}, \infty\right)_{\mathbb{T}}$,

$$
\begin{gathered}
\int_{a}^{\sigma(b)} \eta(s) \Delta \xi(s)=1 \\
\int_{a}^{\sigma(b)} \eta(s) \theta(s) \Delta \xi(s)=\alpha \\
\int_{t_{1}}^{\infty}\left[p^{-1}(\gamma) \int_{\gamma}^{\infty}\left(q(\zeta)+\int_{a}^{\sigma(b)} k(\zeta, s) \Delta \xi(s)\right) \Delta \zeta\right]^{1 / \alpha} \Delta \gamma \\
\varlimsup_{s \rightarrow \infty} \int_{t_{0}}^{s}\left(M(t)-\frac{1}{(\alpha+1)^{\alpha+1}}\right. \\
\left.\times \frac{p(t)\left(\phi^{\Delta}(t)\right)_{+}^{\alpha+1}}{\left[\phi(t) h_{n-2}\left(\delta(t), t_{1}\right) \delta^{\Delta}(t)\right]^{\alpha}}\right) \Delta t=\infty
\end{gathered}
$$

where

$$
\begin{aligned}
M(t)= & \phi(t) q(t) \\
& +\phi(t) \exp \left(\int_{a}^{\sigma(b)} \eta(s) \ln \left(\eta^{-1}(s) k(t, s)\right) \Delta \xi(s)\right), \\
& \left(\phi^{\Delta}(t)\right)_{+}=\max \left\{\phi^{\Delta}(t), 0\right\},
\end{aligned}
$$

then (1) is oscillatory.

Proof. Suppose that (1) has a nonoscillatory solution $x(t)$, then there exists $T_{0}(\in \mathbb{T}) \geqslant t_{0}$ such that $x(t) \neq 0$ for all $t \in\left[T_{0}, \infty\right)_{\mathbb{T}}$. Without loss of generality, we assume that $x(t)>0, x(\delta(t))>0$, and $x(g(t, s))>0$ for $t \in\left[T_{0}, \infty\right)_{\mathbb{T}}$, $s \in[a, b]_{\mathbb{T}_{1}}$, because a similar analysis holds for $x(t)<$ $0, x(\delta(t))<0$ and $x(g(t, s))<0$. From (1) and $\left(H_{2}\right),\left(H_{5}\right)$, we have

$$
\left(p(t)\left|x^{\Delta^{n-1}}(t)\right|^{\alpha-1} x^{\Delta^{n-1}}(t)\right)^{\Delta} \leqslant 0, \quad t \in\left[T_{0}, \infty\right)_{\mathbb{T}} .
$$

Therefore $p(t)\left|x^{\Delta^{n-1}}(t)\right|^{\alpha-1} x^{\Delta^{n-1}}(t)$ is a nonincreasing function and $x^{\Delta^{n-1}}(t)$ is eventually of one sign on $\left[T_{0}, \infty\right)_{\mathbb{T}}$.

We claim that

$$
x^{\Delta^{n-1}}(t)>0 \text { or } x^{\Delta^{n-1}}(t)=0, \quad t \in\left[T_{0}, \infty\right)_{\mathbb{T}} .
$$

Otherwise, if there exists a $t_{1}(\in \mathbb{T}) \geqslant T_{0}$ such that $x^{\Delta^{n-1}}(t)<0$ for $t \in\left[t_{1}, \infty\right)_{\mathbb{T}}$, then from (16), for some positive constant $K$, we have

$$
-p(t)\left(-x^{\Delta^{n-1}}(t)\right)^{\alpha} \leqslant-K, \quad t \in\left[t_{1}, \infty\right)_{\mathbb{T}}
$$

that is

$$
-x^{\Delta^{n-1}}(t) \geqslant\left(\frac{K}{p(t)}\right)^{1 / \alpha}, \quad t \in\left[t_{1}, \infty\right)_{\mathbb{T}},
$$

integrating the above inequality from $t_{1}$ to $t$, we have

$$
x^{\Delta^{n-2}}(t) \leqslant x^{\Delta^{n-2}}\left(t_{1}\right)-K^{1 / \alpha}\left(P(t)-P\left(t_{1}\right)\right) .
$$

Letting $t \rightarrow \infty$, from $\left(H_{2}\right)$, we get $\lim _{t \rightarrow \infty} x^{\Delta^{n-2}}(t)=-\infty$. Analogously, we have $\lim _{t \rightarrow \infty} x^{\Delta^{n-3}}(t)=\lim _{t \rightarrow \infty} x^{\Delta^{n-4}}(t)=$ $\cdots=\lim _{t \rightarrow \infty} x^{\Delta}(t)=\lim _{t \rightarrow \infty} x(t)=-\infty$, which contradicts the fact that $x(t)>0$ for $\left[T_{0}, \infty\right)_{\mathbb{T}}$. Thus, we have proved (17).

So from (16) and (17) and Lemma 5, we obtain

$$
\begin{aligned}
& \left(p(t)\left(x^{\Delta^{n-1}}(t)\right)^{\alpha}\right)^{\Delta} \\
& =p^{\Delta}(t)\left(x^{\Delta^{n-1}}(t)\right)^{\alpha}+p^{\sigma}(t)\left(\left(x^{\Delta^{n-1}}(t)\right)^{\alpha}\right)^{\Delta} \\
& =p^{\Delta}(t)\left(x^{\Delta^{n-1}}(t)\right)^{\alpha} \\
& +p^{\sigma}(t) \alpha\left\{\int _ { 0 } ^ { 1 } \left[(1-h) x^{\Delta^{n-1}}(t)\right.\right. \\
& \left.\left.\quad+h x^{\Delta^{n-1}}(\sigma(t))\right]^{\alpha-1} \mathrm{~d} h\right\} x^{\Delta^{n}}(t)
\end{aligned}
$$

$\leqslant 0$.

Therefore, it follows from the fact $p^{\Delta}(t) \geqslant 0$, we have $x^{\Delta^{n}}(t) \leqslant$ 0 , and from Lemma 1 , there exists an integer $m \in\{1,3, \ldots, n-$ $1\}$ such that (i) and (ii) hold on $\left[T_{0}, \infty\right)_{\mathbb{T}}$. Thus, we have $x^{\Delta}(t)>0$ and then we conclude

$$
\begin{aligned}
& x(t) \geqslant x(\delta(t)) \geqslant x\left(T_{0}\right):=c>0, \\
& x(g(t, s)) \geqslant x(\delta(t)) \geqslant x\left(T_{0}\right):=c>0, \\
& \quad \text { for any } s \in[a, b]_{\mathbb{V}_{1}} .
\end{aligned}
$$

For the case $n=2$, from Lemmas 1 and 2, we get $m=1$. For the case $n \geqslant 4$, we claim that $m=n-1$. Otherwise, we obtain $m \leqslant n-3$, Therefore, it follows from (ii) of Lemma 1 that $x^{\Delta^{n-2}}(t)<0, x^{\Delta^{n-3}}(t)>0$ on $\left[T_{0}, \infty\right)_{\mathbb{T}}$. From $(1)$, we have

$$
\begin{aligned}
\left(p(t)\left(x^{\Delta^{n-1}}(t)\right)^{\alpha}\right)^{\Delta} & \leqslant-q(t) c^{\alpha}-\int_{a}^{\sigma(b)} k(t, s) c^{\theta(s)} \Delta \xi(s) \\
& \leqslant-c_{1}\left(q(t)+\int_{a}^{\sigma(b)} k(t, s) \Delta \xi(s)\right)
\end{aligned}
$$

where

$$
c_{1}= \begin{cases}\min \left\{c^{\alpha}, c^{\theta(b)}\right\}, & 0<c<1, \\ \min \left\{c^{\alpha}, c^{\theta(a)}\right\}, & c \geqslant 1 .\end{cases}
$$


Integrating (23) from $t \geqslant T_{0}$ to $v \geqslant t$ and from (17) we obtain

$$
\begin{aligned}
p(t)\left(x^{\Delta^{n-1}}(t)\right)^{\alpha} \geqslant & p(v)\left(x^{\Delta^{n-1}}(v)\right)^{\alpha} \\
& +c_{1} \int_{t}^{v}\left(q(\zeta)+\int_{a}^{\sigma(b)} k(\zeta, s) \Delta \xi(s)\right) \Delta \zeta \\
\geqslant & c_{1} \int_{t}^{v}\left(q(\zeta)+\int_{a}^{\sigma(b)} k(\zeta, s) \Delta \xi(s)\right) \Delta \zeta .
\end{aligned}
$$

Letting $v \rightarrow \infty$, we have

$$
\begin{aligned}
x^{\Delta^{n-1}}(t) \geqslant & \left(c_{1}\right)^{1 / \alpha} \\
& \times\left[p^{-1}(t) \int_{t}^{\infty}\left(q(\zeta)+\int_{a}^{\sigma(b)} k(\zeta, s) \Delta \xi(s)\right) \Delta \zeta\right]^{1 / \alpha} .
\end{aligned}
$$

Integrating both sides of the last inequality from $T_{0}$ to $t$ and from $x^{\Delta^{n-2}}(t)<0$, we get

$$
\begin{aligned}
- & x^{\Delta^{n-2}}\left(T_{0}\right) \\
\geqslant & x^{\Delta^{n-2}}(t)-x^{\Delta^{n-2}}\left(T_{0}\right) \\
\geqslant & \left(c_{1}\right)^{1 / \alpha} \\
& \quad \times \int_{T_{0}}^{t}\left[p^{-1}(\gamma) \int_{\gamma}^{\infty}\left(q(\zeta)+\int_{a}^{\sigma(b)} k(\zeta, s) \Delta \xi(s)\right) \Delta \zeta\right]^{1 / \alpha} \Delta \gamma .
\end{aligned}
$$

Letting $t \rightarrow \infty$, we get

$$
\begin{gathered}
\int_{T_{0}}^{\infty}\left[p^{-1}(\gamma) \int_{\gamma}^{\infty}\left(q(\zeta)+\int_{a}^{\sigma(b)} k(\zeta, s) \Delta \xi(s)\right) \Delta \zeta\right]^{1 / \alpha} \Delta \gamma \\
\leqslant-x^{\Delta^{n-2}}\left(T_{0}\right)\left(c_{1}\right)^{-1 / \alpha}<\infty
\end{gathered}
$$

which contradicts (13). Thus, we have $m=n-1$, so from Lemma 2 , there exists a sufficiently large $t_{1}(\in \mathbb{T}) \geqslant T_{0}$ such that

$$
x^{\Delta}(\delta(t)) \geqslant h_{n-2}\left(\delta(t), t_{1}\right) x^{\Delta^{n-1}}(\delta(t))>0 \quad \forall t \in\left[t_{1}, \infty\right)_{\mathbb{T}} .
$$

Define

$$
w(t)=\phi(t) \frac{p(t)\left(x^{\Delta^{n-1}}(t)\right)^{\alpha}}{x^{\alpha}(\delta(t))}, \quad \text { for } t \in\left[t_{1}, \infty\right)_{\mathbb{T}} .
$$

Obviously, $w(t)>0$. From (1), $\left(H_{5}\right),(30)$ and $x^{\Delta}(t)>0$, it follows that

$$
\begin{aligned}
w^{\Delta}(t)= & \frac{\phi(t)}{x^{\alpha}(\delta(t))}\left(p(t)\left(x^{\Delta^{n-1}}(t)\right)^{\alpha}\right)^{\Delta} \\
& +\frac{\phi^{\Delta}(t) x^{\alpha}(\delta(t))-\phi(t)\left(x^{\alpha}(\delta(t))\right)^{\Delta}}{x^{\alpha}(\delta(t)) x^{\alpha}(\delta(\sigma(t)))} \\
& \times p(\sigma(t))\left(x^{\Delta^{n-1}}(\sigma(t))\right)^{\alpha} \\
\leqslant & -\phi(t) q(t)-\frac{\phi(t)}{x^{\alpha}(\delta(t))} \\
& \times \int_{a}^{\sigma(b)} k(t, s)(x(g(t, s)))^{\theta(s)} \Delta \xi(s) \\
& +\frac{\phi^{\Delta}(t)}{\phi(\sigma(t))} w(\sigma(t)) \\
& -\frac{\phi(t)\left(x^{\alpha}(\delta(t))\right)^{\Delta} p(\sigma(t))\left(x^{\Delta^{n-1}}(\sigma(t))\right)^{\alpha}}{x^{\alpha}(\delta(t)) x^{\alpha}(\delta(\sigma(t)))} .
\end{aligned}
$$

Now we consider the following two cases.

In the first case $\alpha \geqslant 1$. By $\left(H_{4}\right)$ and Lemmas 4 and 5, we have

$$
\begin{aligned}
& \left(x^{\alpha}(\delta(t))\right)^{\Delta} \\
& \quad=\alpha\left\{\int_{0}^{1}[(1-h) x(\delta(t))+h x(\delta(\sigma(t)))]^{\alpha-1} \mathrm{~d} h\right\}\left(x(\delta(t))^{\Delta}\right. \\
& \quad \geqslant \alpha(x(\delta(t)))^{\alpha-1} x^{\Delta}(\delta(t)) \delta^{\Delta}(t) .
\end{aligned}
$$

From $\left(H_{4}\right),(29)-(32), x^{\Delta}(t)>0, x^{\Delta^{n}}(t) \leqslant 0$, and the fact that

$$
\frac{p^{1 / \alpha}(t) x^{\Delta^{n-1}}(t)}{x(\delta(t))}=\left(\frac{w(t)}{\phi(t)}\right)^{1 / \alpha} \geqslant\left(\frac{w(\sigma(t))}{\phi(\sigma(t))}\right)^{1 / \alpha},
$$

we obtain

$w^{\Delta}(t)$

$$
\begin{aligned}
\leqslant & -\phi(t) q(t)-\frac{\phi(t)}{x^{\alpha}(\delta(t))} \int_{a}^{\sigma(b)} k(t, s)(x(g(t, s)))^{\theta(s)} \Delta \xi(s) \\
& +\frac{\phi^{\Delta}(t)}{\phi(\sigma(t))} w(\sigma(t))-\frac{\phi(t) \alpha x^{\Delta}(\delta(t)) \delta^{\Delta}(t)}{x(\delta(t)) \phi(\sigma(t))} w(\sigma(t)) \\
\leqslant & -\phi(t) q(t)-\frac{\phi(t)}{x^{\alpha}(\delta(t))} \int_{a}^{\sigma(b)} k(t, s)(x(g(t, s)))^{\theta(s)} \Delta \xi(s)
\end{aligned}
$$

$$
\begin{aligned}
& +\frac{\phi^{\Delta}(t)}{\phi(\sigma(t))} w(\sigma(t)) \\
& -\frac{\phi(t) \alpha h_{n-2}\left(\delta(t), t_{1}\right) \delta^{\Delta}(t) x^{\Delta^{n-1}}(t)}{x(\delta(t)) \phi(\sigma(t))} w(\sigma(t))
\end{aligned}
$$




$$
\begin{aligned}
= & -\phi(t) q(t)-\frac{\phi(t)}{x^{\alpha}(\delta(t))} \int_{a}^{\sigma(b)} k(t, s)(x(g(t, s)))^{\theta(s)} \Delta \xi(s) \\
& +\frac{\phi^{\Delta}(t)}{\phi(\sigma(t))} w(\sigma(t))-\frac{\alpha \phi(t) h_{n-2}\left(\delta(t), t_{1}\right) \delta^{\Delta}(t)}{\phi^{(\alpha+1) / \alpha}(\sigma(t)) p^{1 / \alpha}(t)} \\
& \times w^{(\alpha+1) / \alpha}(\sigma(t)) .
\end{aligned}
$$

In the second case $0<\alpha<1$. By $\left(H_{4}\right)$ and Lemmas 4 and 5 , we get

$$
\begin{aligned}
\left(x^{\alpha}(\delta(t))\right)^{\Delta}= & \alpha\left\{\int_{0}^{1}[(1-h) x(\delta(t))+h x(\delta(\sigma(t)))]^{\alpha-1} \mathrm{~d} h\right\} \\
& \times\left(x(\delta(t))^{\Delta}\right. \\
\geqslant & \alpha(x(\delta(\sigma(t))))^{\alpha-1} x^{\Delta}(\delta(t)) \delta^{\Delta}(t) .
\end{aligned}
$$

From $\left(H_{4}\right),(29)-(31),(35), x^{\Delta}(t)>0, x^{\Delta^{n}}(t) \leqslant 0$, $\left(p^{1 / \alpha}(t) x^{\Delta^{n-1}}(t)\right)^{\Delta} \leqslant 0$, and the fact that

$$
\frac{p^{1 / \alpha}(t) x^{\Delta^{n-1}}(t)}{x(\delta(\sigma(t)))} \geqslant \frac{p^{1 / \alpha}(\sigma(t)) x^{\Delta^{n-1}}(\sigma(t))}{x(\delta(\sigma(t)))}=\left(\frac{w(\sigma(t))}{\phi(\sigma(t))}\right)^{1 / \alpha},
$$

we have

$w^{\Delta}(t)$

$$
\begin{aligned}
\leqslant & -\phi(t) q(t)-\frac{\phi(t)}{x^{\alpha}(\delta(t))} \int_{a}^{\sigma(b)} k(t, s)(x(g(t, s)))^{\theta(s)} \Delta \xi(s) \\
& +\frac{\phi^{\Delta}(t)}{\phi(\sigma(t))} w(\sigma(t))-\frac{\alpha \phi(t) h_{n-2}\left(\delta(t), t_{1}\right) \delta^{\Delta}(t)}{\phi^{(\alpha+1) / \alpha}(\sigma(t)) p^{1 / \alpha}(t)} \\
& \times w^{(\alpha+1) / \alpha}(\sigma(t)) .
\end{aligned}
$$

Therefore, for $\alpha>0$, from (34) and (37), we get

$w^{\Delta}(t)$

$$
\begin{aligned}
\leqslant & -\phi(t) q(t)-\frac{\phi(t)}{x^{\alpha}(\delta(t))} \int_{a}^{\sigma(b)} k(t, s)(x(g(t, s)))^{\theta(s)} \Delta \xi(s) \\
& +\frac{\phi^{\Delta}(t)}{\phi(\sigma(t))} w(\sigma(t))-\frac{\alpha \phi(t) h_{n-2}\left(\delta(t), t_{1}\right) \delta^{\Delta}(t)}{\phi^{(\alpha+1) / \alpha}(\sigma(t)) p^{1 / \alpha}(t)} \\
& \times w^{(\alpha+1) / \alpha}(\sigma(t)) .
\end{aligned}
$$

On the other hand, by (11) and (12), we have

$$
\int_{a}^{\sigma(b)} \eta(s)[\theta(s)-\alpha] \Delta \xi(s)=0 .
$$

Therefore, by $\left(H_{4}\right)$, Lemma $7,(39)$, and $x^{\Delta}(t)>0$, we have that for $t \in\left[t_{1}, \infty\right)_{\mathbb{I}}$

$$
\begin{aligned}
& \frac{1}{x^{\alpha}(\delta(t))} \int_{a}^{\sigma(b)} k(t, s)(x(g(t, s)))^{\theta(s)} \Delta \xi(s) \\
& =\int_{a}^{\sigma(b)} k(t, s)\left[\frac{x(g(t, s))}{x(\delta(t))}\right]^{\theta(s)}[x(\delta(t))]^{\theta(s)-\alpha} \Delta \xi(s) \\
& \geqslant \int_{a}^{\sigma(b)} \eta(s) \eta^{-1}(s) k(t, s)[x(\delta(t))]^{\theta(s)-\alpha} \Delta \xi(s) \\
& \geqslant \exp \left(\int_{a}^{\sigma(b)} \eta(s) \ln \left(\eta^{-1}(s) k(t, s)[x(\delta(t))]^{\theta(s)-\alpha}\right) \Delta \xi(s)\right) \\
& =\exp \left(\int_{a}^{\sigma(b)} \eta(s) \ln \left(\eta^{-1}(s) k(t, s)\right) \Delta \xi(s)\right) \\
& \quad \times \exp \left(\ln (x(\delta(t))) \int_{a}^{\sigma(b)} \eta(s)[\theta(s)-\alpha] \Delta \xi(s)\right) \\
& =\exp \left(\int_{a}^{\sigma(b)} \eta(s) \ln \left(\eta^{-1}(s) k(t, s)\right) \Delta \xi(s)\right) .
\end{aligned}
$$

Substituting (40) into (38) we obtain

$$
\begin{aligned}
w^{\Delta}(t) \leqslant & -M(t)+\frac{\phi^{\Delta}(t)}{\phi(\sigma(t))} w(\sigma(t)) \\
& -\frac{\alpha \phi(t) h_{n-2}\left(\delta(t), t_{1}\right) \delta^{\Delta}(t)}{\phi^{(\alpha+1) / \alpha}(\sigma(t)) p^{1 / \alpha}(t)} w^{(\alpha+1) / \alpha}(\sigma(t)) \\
\leqslant & -M(t)+\frac{\left(\phi^{\Delta}(t)\right)_{+}}{\phi(\sigma(t))} w(\sigma(t)) \\
& -\frac{\alpha \phi(t) h_{n-2}\left(\delta(t), t_{1}\right) \delta^{\Delta}(t)}{\phi^{(\alpha+1) / \alpha}(\sigma(t)) p^{1 / \alpha}(t)} w^{(\alpha+1) / \alpha}(\sigma(t)),
\end{aligned}
$$

where $M(t)$ and $\left(\phi^{\Delta}(t)\right)_{+}$are defined by (15).

Taking $a=\left(\phi^{\Delta}(t)\right)_{+} / \phi(\sigma(t)), b=\alpha \phi(t) h_{n-2}(\delta(t)$, $\left.t_{1}\right) \delta^{\Delta}(t) / \phi^{(\alpha+1) / \alpha}(\sigma(t)) p^{1 / \alpha}(t)$, by Lemma 6 and (41), we obtain

$$
w^{\Delta}(t) \leqslant-M(t)+\frac{1}{(\alpha+1)^{\alpha+1}} \frac{p(t)\left(\phi^{\Delta}(t)\right)_{+}^{\alpha+1}}{\left[\phi(t) h_{n-2}\left(\delta(t), t_{1}\right) \delta^{\Delta}(t)\right]^{\alpha}} .
$$


Integrating above inequality (42) from $t_{1}$ to $t>t_{1}$, we have

$$
\begin{aligned}
& w(t) \leqslant w\left(t_{1}\right)-\int_{t_{1}}^{t}\left(M(s)-\frac{1}{(\alpha+1)^{\alpha+1}}\right. \\
& \left.\times \frac{p(s)\left(\phi^{\Delta}(s)\right)_{+}^{\alpha+1}}{\left[\phi(s) h_{n-2}\left(\delta(s), t_{1}\right) \delta^{\Delta}(s)\right]^{\alpha}}\right) \Delta s \\
& \leqslant w\left(t_{1}\right)+\int_{t_{0}}^{t_{1}} M(s) \Delta s \\
& -\int_{t_{0}}^{t}\left(M(s)-\frac{1}{(\alpha+1)^{\alpha+1}}\right. \\
& \left.\times \frac{p(s)\left(\phi^{\Delta}(s)\right)_{+}^{\alpha+1}}{\left[\phi(s) h_{n-2}\left(\delta(s), t_{1}\right) \delta^{\Delta}(s)\right]^{\alpha}}\right) \Delta s .
\end{aligned}
$$

Since $w(t)>0$ for $t>t_{1}$, we have

$$
\begin{gathered}
\int_{t_{0}}^{t}\left(M(s)-\frac{1}{(\alpha+1)^{\alpha+1}} \frac{p(s)\left(\phi^{\Delta}(s)\right)_{+}^{\alpha+1}}{\left[\phi(s) h_{n-2}\left(\delta(s), t_{1}\right) \delta^{\Delta}(s)\right]^{\alpha}}\right) \Delta s \\
\quad \leqslant w\left(t_{1}\right)+\int_{t_{0}}^{t_{1}} M(s) \Delta s-w(t) \leqslant w\left(t_{1}\right)+\int_{t_{0}}^{t_{1}} M(s) \Delta s .
\end{gathered}
$$

Taking upper limit of both sides of the inequality (44) as $t \rightarrow$ $\infty$, the right-hand side is always bounded, which contradicts condition (14). This completes the proof of Theorem 8 .

Theorem 9. Assume that $\left(H_{1}\right)-\left(H_{6}\right)$ and (13) hold for all $t_{1} \in$ $\left[t_{0}, \infty\right)_{\mathbb{T}}$. If there exist a function $\phi(t) \in C_{r d}^{1}\left(\left[t_{0}, \infty\right)_{\mathbb{T}},(0, \infty)\right)$ and a function $\eta(t) \in L_{\xi}[a, b]$ such that $\eta(s)>0$ on $[a, b]_{\mathbb{T}_{1}}$ and (11), (12) hold,

$$
\begin{aligned}
\varlimsup_{s \rightarrow \infty} \int_{t_{0}}^{s}( & \phi(t) q(t)-\frac{1}{(\alpha+1)^{\alpha+1}} \\
& \left.\times \frac{p(t)\left(\phi^{\Delta}(t)\right)_{+}^{\alpha+1}}{\left[\phi(t) h_{n-2}\left(\delta(t), t_{1}\right) \delta^{\Delta}(t)\right]^{\alpha}}\right) \Delta t=\infty
\end{aligned}
$$

where $\left(\phi^{\Delta}(t)\right)_{+}=\max \left\{\phi^{\Delta}(t), 0\right\}$, then (1) is oscillatory.

Proof. The proof is in fact a simpler version of the proof of Theorem 8 . We need only to note that $(\phi(t) /$ $\left.x^{\alpha}(\delta(t))\right) \int_{a}^{\sigma(b)} k(t, s)(x(g(t, s)))^{\theta(s)} \Delta \xi(s)$ is positive in (37).

Theorem 10. Assume that $\left(H_{1}\right)-\left(H_{6}\right)$ and (13) hold for all $t_{1} \in$ $\left[t_{0}, \infty\right)_{\mathbb{T}}$. If there exist a function $\phi(t) \in C_{r d}^{1}\left(\left[t_{0}, \infty\right)_{\mathbb{T}},(0, \infty)\right)$ and a function $\eta(t) \in L_{\xi}[a, b]$ such that $\eta(s)>0$ on $[a, b]_{\mathbb{V}_{1}}$ and (11) and (12) hold,

$$
\varlimsup_{s \rightarrow \infty} \int_{t_{0}}^{s}\left(Q(t)-\frac{p(t)\left(\phi^{\Delta}(t)\right)_{+}}{\left[h_{n-1}\left(\delta(t), t_{1}\right)\right]^{\alpha}}\right) \Delta t=\infty,
$$

where

$$
\begin{aligned}
Q(t)= & \phi(t) q(t) \\
& +\phi(t) \exp \left(\int_{a}^{\sigma(b)} \eta(s) \ln \left(\eta^{-1}(s) k(t, s)\right) \Delta \xi(s)\right), \\
& \left(\phi^{\Delta}(t)\right)_{+}=\max \left\{\phi^{\Delta}(t), 0\right\},
\end{aligned}
$$

then (1) is oscillatory.

Proof. We proceed as in the proof of Theorem 8 to have (30). From (1) and $\left(H_{5}\right),(30)$, Lemmas 3-5, $\left(p(t)\left(x^{\Delta^{n-1}}(t)\right)^{\alpha}\right)^{\Delta} \leqslant 0$, $x^{\Delta^{n}}(t) \leqslant 0$, and $x^{\Delta}(t)>0$, it follows that

$w^{\Delta}(t$

$$
=\frac{\phi(t)}{x^{\alpha}(\delta(t))}\left(p(t)\left(x^{\Delta^{n-1}}(t)\right)^{\alpha}\right)^{\Delta}
$$

$$
+\frac{\phi^{\Delta}(t) x^{\alpha}(\delta(t))-\phi(t)\left(x^{\alpha}(\delta(t))\right)^{\Delta}}{x^{\alpha}(\delta(t)) x^{\alpha}(\delta(\sigma(t)))}
$$$$
\times p(\sigma(t))\left(x^{\Delta^{n-1}}(\sigma(t))\right)^{\alpha}
$$$$
\leqslant-\phi(t) q(t)-\frac{\phi(t)}{x^{\alpha}(\delta(t))} \int_{a}^{\sigma(b)} k(t, s)(x(g(t, s)))^{\theta(s)} \Delta \xi(s)
$$$$
+\frac{\phi^{\Delta}(t)}{x^{\alpha}(\delta(\sigma(t)))} p(t)\left(x^{\Delta^{n-1}}(t)\right)^{\alpha}
$$$$
\leqslant-\phi(t) q(t)-\frac{\phi(t)}{x^{\alpha}(\delta(t))} \int_{a}^{\sigma(b)} k(t, s)(x(g(t, s)))^{\theta(s)} \Delta \xi(s)
$$$$
+\frac{\left(\phi^{\Delta}(t)\right)_{+}}{x^{\alpha}(\delta(t))} p(t)\left(x^{\Delta^{n-1}}(\delta(t))\right)^{\alpha}
$$$$
\leqslant-\phi(t) q(t)-\frac{\phi(t)}{x^{\alpha}(\delta(t))} \int_{a}^{\sigma(b)} k(t, s)(x(g(t, s)))^{\theta(s)} \Delta \xi(s)
$$

$$
+\frac{\left(\phi^{\Delta}(t)\right)_{+} p(t)}{\left[h_{n-1}\left(\delta(t), t_{1}\right)\right]^{\alpha}} .
$$


From (40), we obtain

$$
\begin{aligned}
w^{\Delta}(t) \leqslant & -\phi(t) q(t) \\
& -\phi(t) \exp \left(\int_{a}^{\sigma(b)} \eta(s) \ln \left(\eta^{-1}(s) k(t, s)\right) \Delta \xi(s)\right) \\
& +\frac{\left(\phi^{\Delta}(t)\right)_{+} p(t)}{\left[h_{n-1}\left(\delta(t), t_{1}\right)\right]^{\alpha}} \\
= & -Q(t)+\frac{\left(\phi^{\Delta}(t)\right)_{+} p(t)}{\left[h_{n-1}\left(\delta(t), t_{1}\right)\right]^{\alpha}} .
\end{aligned}
$$

Integrating this inequality from $t_{1}$ to $t>t_{1}$, we have

$$
\begin{aligned}
w(t) \leqslant & w\left(t_{1}\right)-\int_{t_{1}}^{t}\left(Q(s)-\frac{\left(\phi^{\Delta}(s)\right)_{+} p(s)}{\left[h_{n-1}\left(\delta(s), t_{1}\right)\right]^{\alpha}}\right) \Delta s \\
\leqslant & w\left(t_{1}\right)+\int_{t_{0}}^{t_{1}} Q(s) \Delta s \\
& -\int_{t_{0}}^{t}\left(Q(s)-\frac{\left(\phi^{\Delta}(s)\right)_{+} p(s)}{\left[h_{n-1}\left(\delta(s), t_{1}\right)\right]^{\alpha}}\right) \Delta s .
\end{aligned}
$$

Taking upper limit of both sides of the inequality (50) as $t \rightarrow$ $\infty$ and using (46) we obtain a contradiction to the fact that $w(t)>0$ on $\left[t_{1}, \infty\right)_{\mathbb{T}}$. This completes the proof of Theorem 9.

Theorem 11. Assume that $\left(H_{1}\right)-\left(H_{6}\right)$ and (13) hold for all $t_{1} \in$ $\left[t_{0}, \infty\right)_{\mathbb{T}}$. If there exist a function $\phi(t) \in C_{r d}^{1}\left(\left[t_{0}, \infty\right)_{\mathbb{T}},(0, \infty)\right)$ and a function $\eta(t) \in L_{\xi}[a, b]$ such that $\eta(s)>0$ on $[a, b]_{\mathbb{V}_{1}}$ and (11), (12) hold,

$$
\varlimsup_{s \rightarrow \infty} \int_{t_{0}}^{s}\left(\phi(t) q(t)-\frac{p(t)\left(\phi^{\Delta}(t)\right)_{+}}{\left[h_{n-1}\left(\delta(t), t_{1}\right)\right]^{\alpha}}\right) \Delta t=\infty,
$$

where $\left(\phi^{\Delta}(t)\right)_{+}=\max \left\{\phi^{\Delta}(t), 0\right\}$, then (1) is oscillatory.

Proof. The proof is in fact a simpler version of the proof of Theorem 10. We need only to note that $\phi(t) \exp \left(\int_{a}^{\sigma(b)} \eta(s) \ln \left(\eta^{-1}(s) k(t, s)\right) \Delta \xi(s)\right)$ is positive in (49).

Remark 12. If we let $\alpha$ be the ratio of positive odd integers, $f(t, x(\delta(t)))=q(t)(x(t))^{\alpha}, k(x, s)=0$, and $\delta(t)=t$ and use the convention that $\ln 0=-\infty, e^{-\infty}=0$, then Theorem 8 reduces to [9, Theorems 2.3] and Theorem 10 reduces to [9, Theorems 2.2].

\section{Conflict of Interests}

The authors declare that there is no conflict of interests regarding the publication of this paper.

\section{Acknowledgments}

This research was supported by the National Natural Science Foundations of China (nos. 11171178 and 61104136), the Natural Science Foundation of Shandong Province of China (no. ZR2010FQ002), and the Foundation of Qufu Normal University (no. XJ201014).

\section{References}

[1] S. R. Grace, "On the oscillation of $n$th order dynamic equations on time-scales," Mediterranean Journal of Mathematics, vol. 10, no. 1, pp. 147-156, 2013.

[2] S. R. Grace, R. P. Agarwal, and A. Zafer, "Oscillation of higher order nonlinear dynamic equations on time scales," Advances in Difference Equations, vol. 2012, article 67, 2012.

[3] D. X. Chen, "Oscillation and asymptotic behavior for $n$ th-order nonlinear neutral delay dynamic equations on time scales," Acta Applicandae Mathematicae, vol. 109, no. 3, pp. 703-719, 2010.

[4] R. Mert, "Oscillation of higher-order neutral dynamic equations on time scales," Advances in Difference Equations, vol. 2012, article 68, 2012.

[5] L. Erbe, B. Jia, and A. Peterson, "Oscillation of $n$th order superlinear dynamic equations on time scales," The Rocky Mountain Journal of Mathematics, vol. 41, no. 2, pp. 471-491, 2011.

[6] B. Karpuz, "Asymptotic behaviour of bounded solutions of a class of higher-order neutral dynamic equations," Applied Mathematics and Computation, vol. 215, no. 6, pp. 2174-2183, 2009.

[7] B. Karpuz, "Unbounded oscillation of higher-order nonlinear delay dynamic equations of neutral type with oscillating coefficients," Electronic Journal of Qualitative Theory of Differential Equations, vol. 9, pp. 1-14, 2009.

[8] D. X. Chen and P. X. Qu, "Oscillation of even order advanced type dynamic equations with mixed nonlinearities on time scales," Journal of Applied Mathematics and Computing, vol. 44, no. 1-2, pp. 357-377, 2014

[9] S. R. Grace, "On the oscillation of higher order dynamic equations," Journal of Advanced Research, vol. 4, pp. 201-204, 2013.

[10] L. Erbe, B. Karpuz, and A. C. Peterson, "Kamenev-type oscillation criteria for higher-order neutral delay dynamic equations," International Journal of Difference Equations, vol. 6, no. 1, pp. 1-16, 2011.

[11] T. Sun, H. Xi, and W. Yu, "Asymptotic behaviors of higher order nonlinear dynamic equations on time scales," Journal of Applied Mathematics and Computing, vol. 37, no. 1-2, pp. 177-192, 2011.

[12] T. X. Sun, H. J. Xi, and X. F. Peng, "Asymptotic behavior of solutions of higher-order dynamic equations on time scales," Advances in Difference Equations, vol. 2011, Article ID 237219, 14 pages, 2011.

[13] Z. G. Zhang, W. L. Dong, Q. L. Li, and H. Y. Liang, "Existence of nonoscillatory solutions for higher order neutral dynamic equations on time scales," Journal of Applied Mathematics and Computing, vol. 28, no. 1-2, pp. 29-38, 2008.

[14] S. Hilger, "Analysis on measure chains-a unified approach to continuous and discrete calculus," Results in Mathematics, vol. 18, no. 1-2, pp. 18-56, 1990.

[15] M. Bohner and A. Peterson, Dynamic Equations on Time Scales, Birkhäuser, Boston, Mass, USA, 2001. 
[16] R. P. Agarwal and M. Bohner, "Basic calculus on time scales and some of its applications," Results in Mathematics, vol. 35, no. 1-2, pp. 3-22, 1999.

[17] D. X. Chen, "Oscillation of second-order Emden-Fowler neutral delay dynamic equations on time scales," Mathematical and Computer Modelling, vol. 51, no. 9-10, pp. 1221-1229, 2010.

[18] G. H. Hardy, J. E. Littlewood, and G. Pólya, Inequalities, Cambridge University Press, Cambridge, UK, 2nd edition, 1952.

[19] Y. Sun, "Interval oscillation criteria for second-order dynamic equations with nonlinearities given by Riemann-Stieltjes integrals," Abstract and Applied Analysis, vol. 2011, Article ID 719628, 14 pages, 2011. 


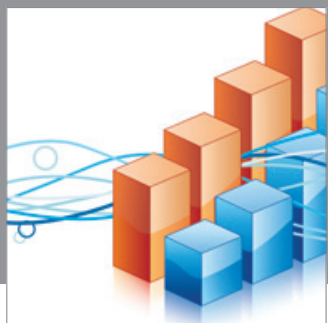

Advances in

Operations Research

mansans

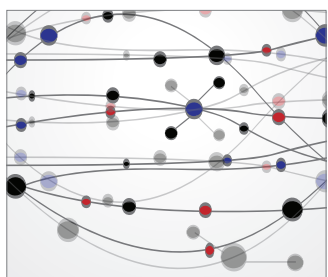

The Scientific World Journal
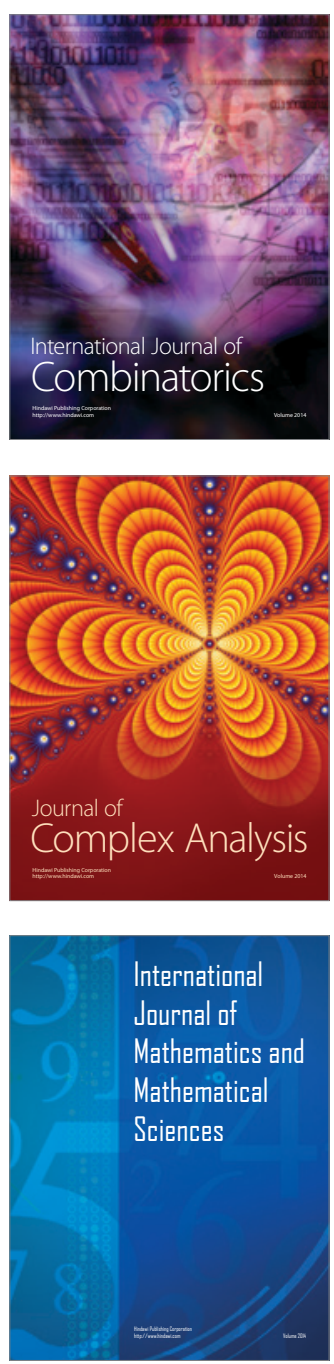
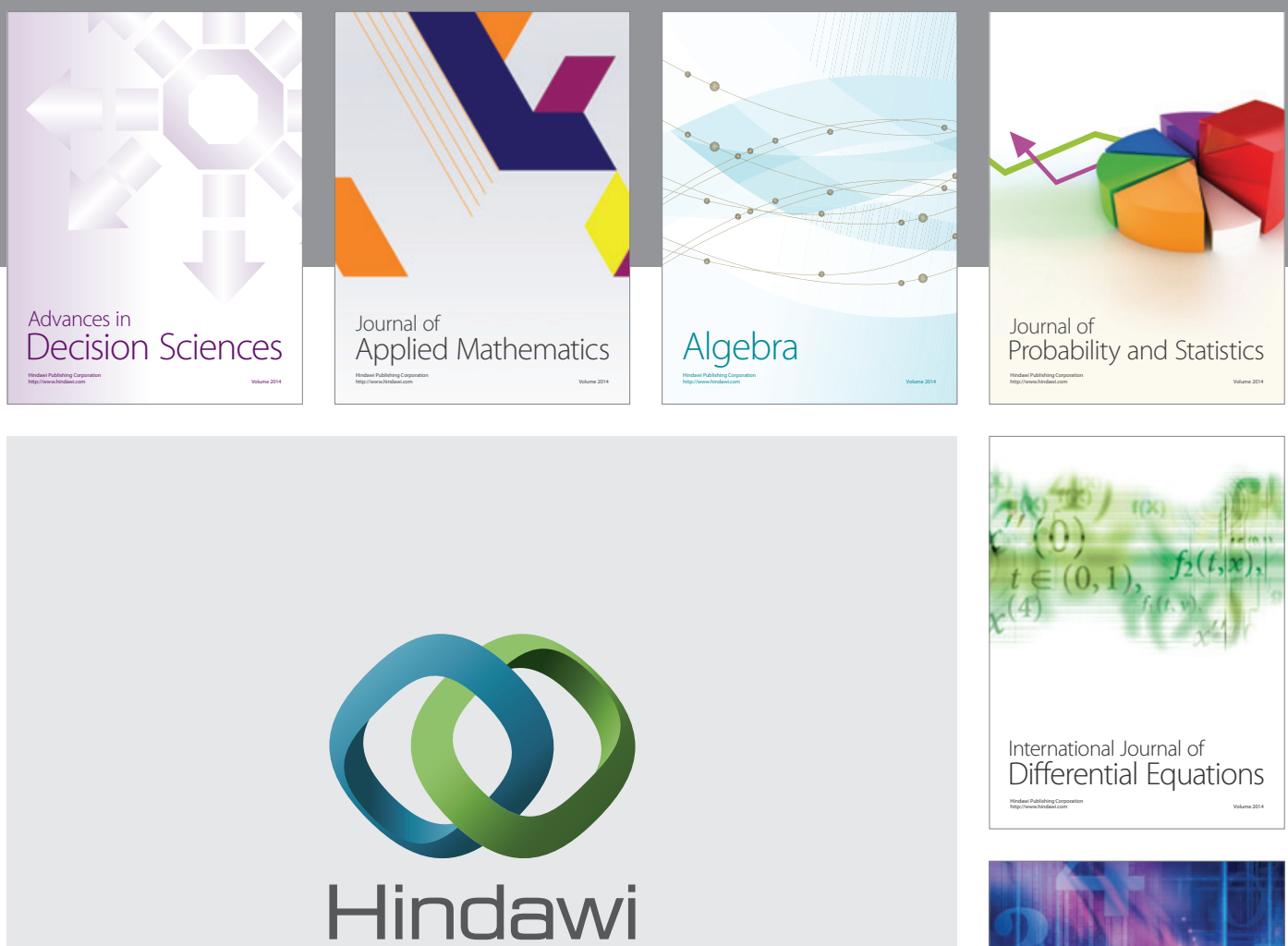

Submit your manuscripts at http://www.hindawi.com
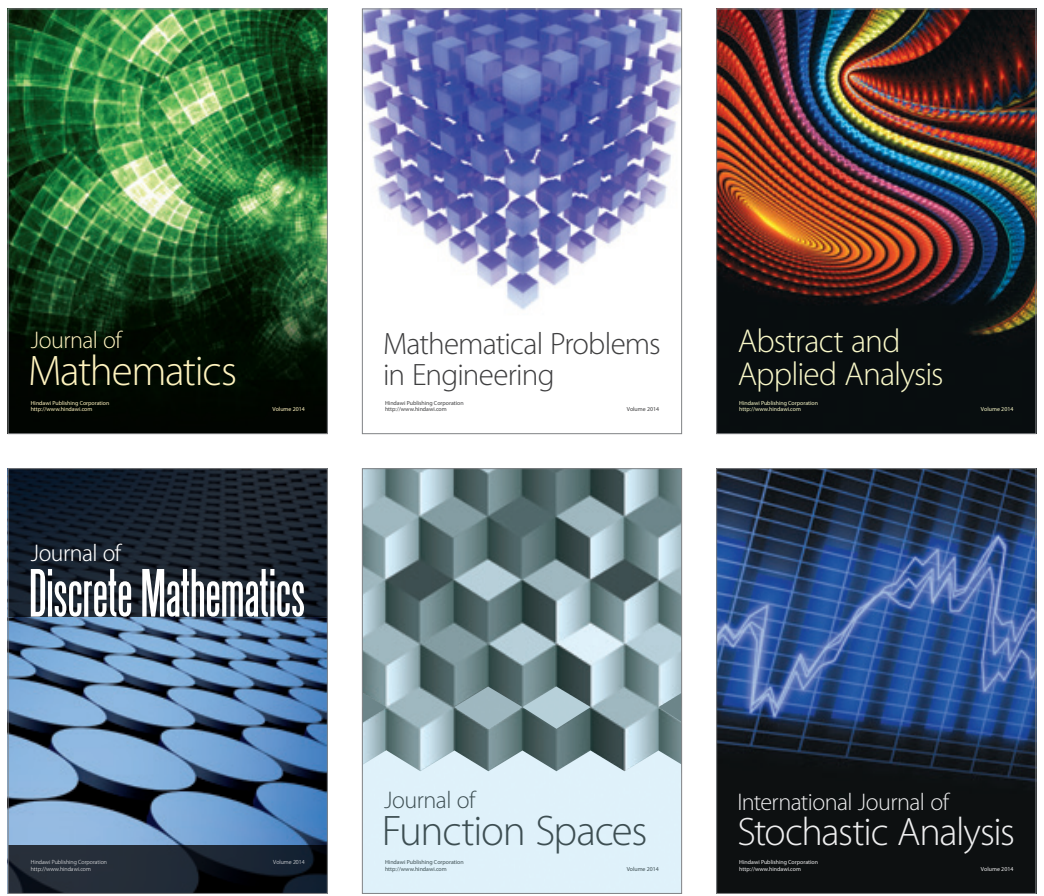

Journal of

Function Spaces

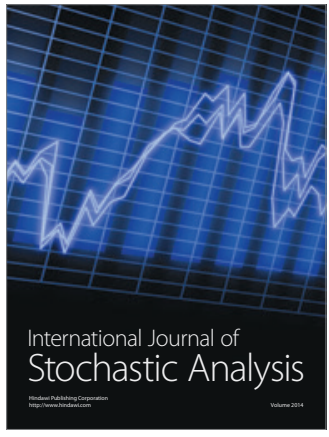

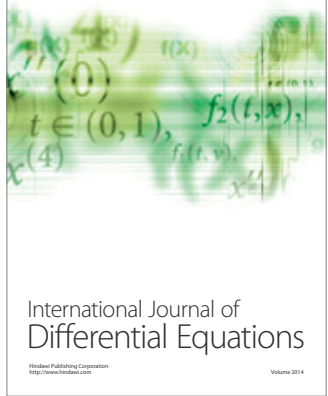
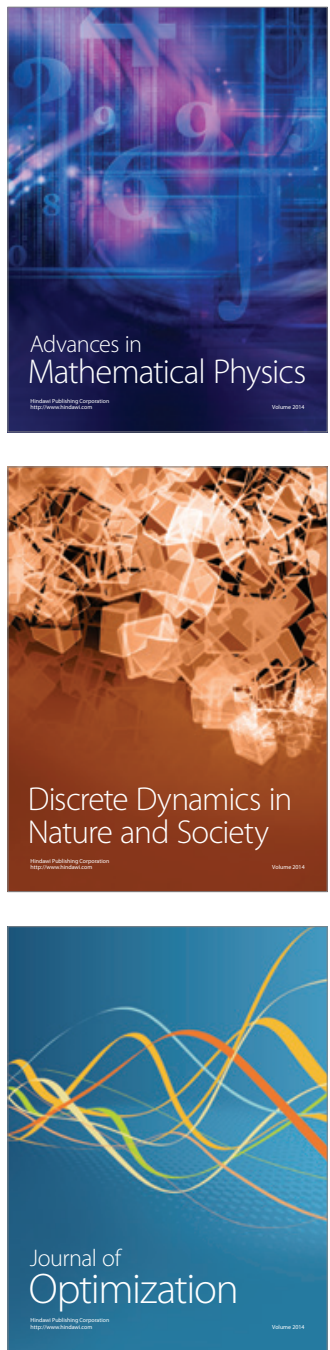